\title{
Active surveillance of antibiotic resistance patterns in urinary tract infections in primary care in Switzerland
}

Plate, Andreas ; Kronenberg, Andreas ; Risch, Martin ; Mueller, Yolanda ; Di Gangi, Stefania ; Rosemann, Thomas ; Senn, Oliver

\begin{abstract}
PURPOSE: Urinary tract infections (UTI) are one of the most common reasons for prescribing antibiotics in primary care. In Switzerland, the Swiss Center for Antibiotic Resistances (ANRESIS) provides resistance data by passive surveillance, which overestimates the true resistance rates. The aim of this study was to provide actual data of the antimicrobial resistance patterns in patients with UTI in Swiss primary care. METHODS: From June 2017 to August 2018, we conducted a cross-sectional study in 163 practices in Switzerland. We determined the resistance patterns of uropathogens in patients with a diagnosis of a lower UTI and analyzed risk factors for resistance. Patients with age < 18 years, pregnancy or a pyelonephritis were excluded. RESULTS: 1352 patients (mean age 53.8, 94.9\% female) were included in the study. 1210 cases (89.5\%) were classified as uncomplicated UTI. Escherichia coli (E. coli) was the most frequent pathogen (74.6\%). Susceptibility proportions of E. coli to ciprofloxacin (88.9\%) and trimethoprim-sulfamethoxazol (TMP/SMX) (85.7\%) were significantly higher than the proportions reported by ANRESIS. We found high susceptibility to the recommended first-line antibiotics nitrofurantoin (99.5\%) and fosfomycin (99.4\%). Increasing age, antimicrobial exposure and a recent travel history were independently associated with resistance. DISCUSSION: In this study, we report actual data on the resistance patterns of uropathogens in primary care in Switzerland. Escherichia coli showed low resistance rates to the recommended first-line antibiotics. Resistance to TMP/SMX was significantly lower than reported by ANRESIS, making TMP/SMX a suitable and cheap alternative for the empirical treatment.
\end{abstract}

DOI: https://doi.org/10.1007/s15010-019-01361-y

Posted at the Zurich Open Repository and Archive, University of Zurich

ZORA URL: https://doi.org/10.5167/uzh-178699

Journal Article

Accepted Version

Originally published at:

Plate, Andreas; Kronenberg, Andreas; Risch, Martin; Mueller, Yolanda; Di Gangi, Stefania; Rosemann, Thomas; Senn, Oliver (2019). Active surveillance of antibiotic resistance patterns in urinary tract infections in primary care in Switzerland. Infection, 47(6):1027-1035.

DOI: https://doi.org/10.1007/s15010-019-01361-y 
1 Title: Active surveillance of antibiotic resistance patterns in urinary tract infections in primary care in Switzerland.

2 Authors: Andreas Plate ${ }^{1}$, Andreas Kronenberg ${ }^{2}$, Martin Risch ${ }^{3}$, Yolanda Mueller ${ }^{4}$, Stefania Di Gangi ${ }^{1}$, Thomas

3 Rosemann ${ }^{1}$, Oliver Senn ${ }^{1}$

4 1: Institute of Primary Care, University and University Hospital of Zurich, Zurich, Switzerland.

5 2: Swiss Center for Antibiotic Resistance, Institute for Infectious Diseases, University Bern, Bern and Medix

6 General Practice Network, Bern, Switzerland

7 3: labormedizinisches zentrum Dr Risch Ostschweiz AG, Buchs, St. Gallen

8 4: Department of Family Medicine, Center for Primary Care and Public Health (Unisanté), University of Lausanne,

9 Switzerland

Contact Address:

Andreas Plate, MD

Institute of Primary Care, University of Zurich

Pestalozzistrasse 24

8091 Zurich, Switzerland

Phone: +41 442559855

Fax: +41442559097

e-Mail: andreas.plate@usz.ch

Running title: Antibiotic resistance patterns in primary care in Switzerland

Key words: urinary tract infection, resistance rates, susceptibility rates, E. coli, primary care, Switzerland 
Funding: This study was supported by grants from SwissLife, Blumenau-Léonie Hartmann Foundation, and

25 Innova Foundation. ANRESIS is funded by the Swiss federal office of public health and the University of Bern. They had no influence over the study design, study results, interpretation of the data and publication.

Acknowledgement: We wish to thank primary care physicians and patients that participated in the study; we acknowledge Swiss family medicine institutes in Geneva, Lausanne, Lucerne, Basel and Zürich, the representatives of the local medical networks that supported recruitment of family physicians and the anresislaboratories for sharing their data; we acknowledge Dr. phil. II Michael Ritzler und Dr. scient. med. Nadia

32 Wohlwend as well as all involved laboratory members of the laboratory Risch, which performed all urine analysis for this study. 


\section{Abstract:}

Purpose: Urinary tract infections (UTI) are one of the most common reasons for prescribing antibiotics in primary care. In Switzerland, the Swiss Center for Antibiotic Resistances (ANRESIS) provides resistance data by passive surveillance, which overestimates the true resistance rates. The aim of this study was to provide actual data of the antimicrobial resistance patterns in patients with UTI in Swiss primary care.

Methods: From June 2017 to August 2018, we conducted a cross-sectional study in 163 practices in Switzerland. We determined the resistance patterns of uropathogens in patients with a diagnosis of a lower UTI and analysed risk factors for resistance. Patients with age $<18$ years, pregnancy or a pyelonephritis were excluded.

Results: 1,352 patients (mean age 53.8, 94.9\% female) were included in the study. 1,210 cases $(89.5 \%)$ were classified as uncomplicated UTI. E. coli was the most frequent pathogen (74.6\%). Susceptibility proportions of $E$. coli to ciprofloxacin (88.9\%) and trimethoprim-sulfamethoxazol (TMP/SMX) (85.7\%) were significantly higher than the proportions reported by ANRESIS. We found high susceptibility to the recommended first line antibiotics nitrofurantoin (99.5\%) and fosfomycin (99.4\%). Increasing age, antimicrobial exposure and a recent travel history were independently associated with resistance.

Discussion: In this study, we report actual data on the resistance patterns of uropathogens in primary care in Switzerland. E. coli showed low resistance rates to the recommended first line antibiotics. Resistance to TMP/SMX was significantly lower than reported by ANRESIS, making TMP/SMX a suitable and cheap alternative for the empirical treatment. 


\section{Introduction}

54

Urinary tract infections (UTI) are one of the most common infections worldwide [1] and almost every second women will have at least one episode during lifetime [2]. Although UTIs may be self-limiting, UTIs are one of the most common reasons for prescribing antibiotics in primary care [3]. A progress to an upper UTI /pyelonephritis is rare [4], but seem to be more common without antibiotic therapy. Furthermore, antibiotic therapy results in a faster symptom relief compared to placebo or anti-inflammatory therapies [5, 6]. Most guidelines do not recommend a microbiological diagnosis in cases of an uncomplicated UTI (uUTI) before treatment $[7,8]$. The choice of the empirical antibiotic treatment depends on the expected bacteria and their antibiotic resistance patterns. To date fosfomycin, nitrofurantoin, pivmecillinam and trimethoprim-sulfamethoxazol (TMP/SMX) were recommended as empirical first line therapy in most guidelines. The use of TMP/SMX is limited to areas, where local resistance rates are known and where they do not exceed 20\% [7]. In Switzerland, the Swiss Center for Antibiotic Resistances (www.anresis.ch) provides a nationwide passive surveillance [9]. However, because in cases of an uUTI no microbiological diagnostic is done in most cases, passive surveillance may overestimate the resistance prevalence in the community setting [10]. The aim of this study was to provide actual data of the antimicrobial resistance patterns in patients with a lower UTI in primary care. 
70

71

This cross-sectional study was conducted from June 2017 to August 2018 in 161 Swiss primary care practices as well as in two large "walk-in" practices. The general practitioners (GP) proposed study participation to all patients aged 18 and older with a clinical diagnosis of a lower UTI in consecutive order. Pregnant women and patients with a pyelonephritis were excluded. No patient could be included twice in the study in case of recurrence or treatment failure. Diagnostic criteria of an UTI (complicated and uncomplicated) were provided to all GPs to ensure diagnostic standardization [8]. UTI was defined as the new onset of typical symptoms (dysuria, pollakiuria, urgency or haematuria) and a positive urine dipstick (positive leucocytes). Uncomplicated cystitis was defined as cystitis in otherwise healthy women without the history or the clinical suspicion of any functional or anatomical abnormalities of the urinary tract. UTIs in male patients or in female patients with concomitant (urological) disorders (according to Swiss national guidelines [8]) were considered as complicated. In case of study participation and signed informed consent, a urine specimen was collected for microbiological analysis. Furthermore, epidemiological and clinical data were recorded. Finally, the GP had to determine the final diagnosis (uncomplicated or complicated cystitis), and whether they would have done a microbiological analysis apart of the study.

Resistance data from ANRESIS (2018 resistance data) were acquired by using the following selection criteria: All urinary Escherichia coli (E. coli) isolates from adult (age >15) outpatients (private physicians, ambulatories or emergency departments), (assessed 17.06.2019) (www.anresis.ch). Comparisons of regional susceptibility patterns were done according to the geographical classification of Switzerland done by ANRESIS.

Microbiological analysis:

Urine samples for culture were collected in a sterile container containing boric acid as a preservative. The urine was plated onto a chromogenic and blood agar medium and an inhibition test for detection of a possible antibiotic pretreatment was performed. After $24 / 48 \mathrm{~h}$ incubation time, positive culture was defined as growth of $10^{\wedge} 3$ colony forming units or more. Bacteria were subjected to an identification procedure by mass spectrometry (MALDI TOF) and to an automated antimicrobial susceptibility testing (Vitek 2). Intermediate resistance was handled as resistant for resistance analysis.

Ethics: 
The study was approved by the local ethics committee (BASEC Number: 2016-01918) and every patient signed a study specific informed consent.

Analysis:

Summary statistics were reported as means (standard deviation, SD), and number (percentage, \%) as appropriate. Patient characteristics and microbiological results were compared between cUTI and uUTI; Independent sample Student's $t$ test was used for continuous variables and chi-square or Fisher's test, as appropriate, were used for categorical variables. Antibiotic susceptible rates of $E$. coli were compared to the rates provided by Swiss passive surveillance using the Chi squared test with simulated p-values, computed by a Monte Carlo test with 2000 replicates. The $95 \%$ confidence interval (CI) for the susceptible proportions were reported, too. Resistance to Fluoroquinolones (FC) means resistance to at least one out of ciprofloxacin, levofloxacin, moxifloxacin, or norfloxacin. Univariable and multivariable logistic regression models were performed to identify the association between patient characteristics, together with type of UTI, and antibiotics resistance rate of E.coli. Only women infected with $E$. coli were considered in the regression analysis. The study's exploratory nature required nonparsimonious multivariable regression models to identify variables for further exploration in future studies. These models were performed using automatic stepwise selection estimation with likelihood ratio testing (P-value $\leq 0.20)$ specified as the test of significance to include or exclude variables. For all other tests, $\mathrm{P} \leq 0.05$ was considered statistically significant. As sensitivity analysis, we estimated the intracluster correlation coefficient (ICC), using a mixed regression model with a random effect at GP level. All analyses were carried out using statistical package R, R Core Team (2016). (R: A language and environment for statistical computing. R Foundation for Statistical Computing, Vienna, Austria. URL https://www.R-project.org/).

\section{Results}

1,454 patients were screened for study participation. 33 patients refused to participate. In total 1,421 urine samples were collected (Figure 1). 69 patients were excluded due to missing leukocyturia (46), age $<18$ (10), absence of classical symptoms (9), pregnancy (2), or other reasons (2). Finally, 1,352 urine samples were included in the overall analysis. Basic demographic and clinical information are shown in Table 1. 1,210 cases (89.5\%) were reported as uUTI and 129 as cUTI (9.5\%). Thirteen cases (1\%) resulted unclassified, hence these cases were excluded for comparison analysis, but still included in the overall analysis. $94.9 \%$ of the participants were female. The overall mean age was 53.75 (standard deviation: 20.8) years. 


\section{Microbiological analysis}

Urine cultures were positive in $87.1 \%$ (1,117 cases) (Table 2). In 143 cases (12.1\%) two pathogens were detected and 140 cases $(11.9 \%)$ were considered as contaminated due to growth of 3 or more pathogens and were excluded from further analysis. Gram-negative rods were present in $86.8 \%$, enterococci in $6.9 \%$ and other gram-positive bacteria in $19.4 \%$ of all cases. However, growth of only gram-negative rods, enterococci or other gram-positive bacteria in a culture was detected in $72.7 \%, 3.2 \%$ and $8.6 \%$, respectively. E. coli was the most common pathogen and was found in $74.6 \%$ of all cases. Comparing the causing uropathogens in the uUTI and cUTI, there were no significant differences.

\section{Resistance proportions}

Resistance proportions were reported for E. coli (additional resistance proportions for Klebsiella spp.,

Enterobacter spp. and Proteus spp. were provided in Supplemental Table 4). E. coli showed high susceptibility rates to the recommended first line antibiotics fosfomycin $(99.35 \%, 95 \%$ confidence interval [CI]: $99.34 \%$ 99.37\%), nitrofurantoin (99.48\%, 95\% CI: 99.47\%-99.5\%), and TMP/SMX (85.66\%, 95\% CI: 85.58\%-85.74\%). With exception of the Geneva area, resistance proportions to TMP/SMX were below $20 \%$ in all areas of Switzerland. Although not statistically significant, we observed the lowest susceptibility proportions against TMP/SMX in the French-speaking western areas of Switzerland. 88.89\% (95\% CI: 88.82\%-88.96\%) of the isolates were susceptible to ciprofloxacin (Table 3) and $85.40 \%$ (95\% CI: 85.32\%-85.48\%) of the isolates were susceptible to norfloxacin. In comparison to the data provided by ANRESIS (passive surveillance), we found significantly higher susceptibility proportions of $E$. coli to both TMP/SMX and ciprofloxacin ( $\mathrm{p}<0.001$ in both groups). There were no significant differences in the susceptibility to fosfomycin and nitrofurantoin $(\mathrm{p}=0.14$, and $\mathrm{p}=0.543$, respectively) between active and passive surveillance. With the exception of nitrofurantoin ( $\mathrm{p}=0.011)$, susceptible proportions to the antibiotics did not differ significantly between the different regions across Switzerland in our study. Nitrofurantoin susceptibility was reduced in the south region of Switzerland (susceptibility rate 90.9\%), compared to the other regions (susceptibility rates $>97.3 \%$ ). However, this finding could be biased due to the very limited specimen numbers in the south region $(\mathrm{n}=11)$.

Comparing the susceptibility proportions of $E$. coli in uUTI and cUTI, we found no significant differences (Supplemental Table 1). Furthermore, there was no significant difference in the susceptibility proportion to TMP/SMX in patients in which the GP would have done a urine analysis apart from the study, compared to the 
patients without a urine analysis. In contrast, susceptibility to ciprofloxacin was significant lower in patients in which the GP would have done a urine analysis apart from the study (Supplemental Table 2).

\section{Risk factors for $E$. coli resistance}

Univariate and multivariate logistic regression analysis for identifying variables as risk factors for antibiotic resistance to the recommended first line antibiotics (TMP/SMX, nitrofurantoin, and fosfomycin) or to FC in women infected with E. coli are shown in Table 4. Additional analysis for each antibiotic in separate were provided in Supplemental Table 3. Clustering by GP practice was negligible, with ICC 0.012 for resistance to first-line antibiotics.

Analysing risk factors for resistance to any recommended first line antimicrobial agent, antibiotic exposure for any reason within the last 3 months and a recent travel to Africa were associated with an increased risk of resistance, which is also true for resistance to TMP/SMX alone. Compared to antimicrobial exposure for any reason, antimicrobial exposure specific for an UTI was not associated with an increased resistance. Age, a recent hospital stay, and a travel history to Oceania were associated with increased risk of resistance to fosfomycin, whereas no risk factors could be identified for resistance to nitrofurantoin. Resistance to FC was associated with age, living in long-term care, antimicrobial exposure and traveling within Europe and Asia.

\section{Discussion}

In this study, we determined the frequency and susceptibility proportions of uropathogens in urinary tract infections in primary care in Switzerland. We found high susceptibility proportions of E. coli to the recommended first line antibiotics and higher susceptibility proportions for TMP/SMX and ciprofloxacin as reported by the Swiss national passive surveillance.

E. coli is the most frequent pathogen in both uncomplicated and complicated UTI [11] and accounts for up to 95\% of urinary tract infections [7] as also seen in our study. Knowledge of local resistance patterns of E.coli is therefore crucial for the consideration of an adequate empirical treatment. In our cohort, clinical significant growth, after excluding contaminated samples, could be detected in $76 \%$, which is similar to other studies $[12,10]$. E.coli was the most frequent pathogen and showed high susceptibility proportions to the recommended first line antibiotics fosfomycin, nitrofurantoin, and TMP/SMX. Current guidelines recommend the use of TMP/SMX only if local susceptibility proportions exceed $80 \%$. With exception of the Geneva area, this premise is fulfilled in all geographic areas of Switzerland. Although not statistically significant, we observed a trend for higher resistance proportions against TMP/SMX in the French-speaking western areas of Switzerland. This could be explained by 
the fact that the highest antibiotic prescription rates are also seen the French-speaking parts of Switzerland [13, 14] and an association between resistance rates and antibiotic prescription is frequently reported in the literature $[15,14]$.

Compared to the Swiss national passive surveillance, susceptibility proportions to TMP/SMX and ciprofloxacin were significantly higher in our cohort. This reflects the selection bias in passive surveillance systems, as microbiological analysis is not recommended in most outpatients with a uUTI and higher resistance rates are seen in patients with a cUTI [16]. Susceptibility proportions of nitrofurantoin and fosfomycin did not differ significantly from the reported proportions by ANRESIS. This is due to overall high susceptibility proportions (>98\%) inboth our cohort and ANRESIS.

10 years ago, a similar study investigated the resistance proportions in around 1,000 outpatient UTI cases in the canton of Berne in Switzerland [10]. The reported susceptibility proportions of E. coli to TMP/SMX (71\% to 80\%) were lower than the susceptibility proportions in our study. The same is true for nitrofurantoin, with higher susceptibility proportions in our study. Resistance proportions to fosfomycin were similar in both studies. It seems that at least in the outpatient setting there is no deterioration of resistance patterns against fist line antimicrobial agents within the last decade, which is remarkable as the frequency of resistant uropathogens is steadily increasing $[17,16]$. One the other hand, susceptibility to norfloxacin (the only reported FC), was reported higher compared to our study.

It is generally considered, that susceptibility rates of uropathogens in uUTI are higher compared to cUTI $[7,12$, 18-21] and that E.coli is the most common uropathogen in both conditions, despite the wider microbiological spectrum in cUTI [22]. As expected in our study E. coli was the most frequent pathogen in both uUTI and cUTI (74.8\% and $72.3 \%$, respectively), but susceptibility proportions did not differ significantly between both groups. These findings could be explained by two reasons: First, the numbers of cUTI were relative low $(<10 \%)$. Second, in contrast to the well-known and accepted definition of uUTI, the definition for cUTI is more heterogeneous. There is evidence, that for example in young men a UTI can be uncomplicated [23]. The microbiological patterns of causing pathogens in the different aetiologies of cUTI are unknown. Our findings, that there can be similarities in the resistance patterns and in the causing pathogens in both groups, support the need for a more detailed stratification and treatment recommendations of cUTI [11,23] (at least for ambulatory care). National guidelines recommend a microbiological culture and the use of antibiotics with a good prostate penetration in UTI in men [8]. Both TMP/SMX and FC have an excellent prostate penetration. Due to the similar resistance rates in uUTI and cUTI in our cohort, empirical therapy with TMP/SMX seems to be feasible also in men until resistance analysis 
is available. According to pharmacological data also fosfomycin has an good penetration into the prostate [24],

216 but today fosfomycin is not routinely used in the treatment of an prostatitis [22]. In contrast, nitrofurantoin does not penetrate into prostatic tissue adequately [25].

Multivariable regression analysis revealed age, prior antibiotic exposure and a recent travel history as risk factors 29] and these factors need to be considered in the choice of the empiric treatment.

Strengths/limitations: In this prospective trial, we sampled urine specimen across all regions in Switzerland and all specimen were analysed in one central laboratory. The overall numbers of patients, which refused to participate and the exclusions were low (Figure 1), indicating a low risk of selection bias, assuming the data are representative for primary care in Switzerland; nevertheless certain subgroup analysis have to be interpreted with caution due to small sample sizes (e.g. comparisons across regions, multivariable modelling) and limited data (reason for classification uUTI vs. cUTI).

Current national and international guidelines recommend fosfomycin or nitrofurantoin for the empirical treatment of uUTI. Depending on the local resistance rate, TMP/SMX is an additional first line antimicrobial agent. In our cohort, we could report resistance proportions below 20\% to TMP/SMX in nearly all areas of Switzerland. Thus, TMP/SMX remains a suitable antibiotic for the empirical treatment of uUTI and even cUTI in primary care in Switzerland, especially as the treatment costs of the standard regime TMP/SMX are similar to that of nitrofurantoin, but three times cheaper compared to fosfomycin. However, in patients with a systemic antibiotic exposure within the last three months or a travel history to Africa, clinicians should prefer a therapy with nitrofurantoin or fosfomycin, Of note, a recent multicentric study showed superiority of nitrofurantoin over fosfomycin [30]. of UTIs and were recommended for the empirical therapy for many years. Despite UTIs, FC are important in the treatment in extra- urogenital infections like intra-abdominal infections, soft tissue, and bone and joint infections.

240 Due to excessive use, increasing resistance rates not only in uropathogens were observed [7]. Increasing FC resistance rates are a serious public health treat [16], in addition to the general potential side effects like ecological damage to gut flora or tendinopathies. Therefore, we support the recommendations of current guidelines to avoid 
245 In conclusion, we could show low resistance patterns to the recommended first line antibiotics fosfomycin,

246 nitrofurantoin and TMP/SMX in both uncomplicated and complicated UTI in primary care in Switzerland.

247

248 Conflict of interest: All authors declare no conflict of interest.

249

250 
References

1. Foxman B. The epidemiology of urinary tract infection. Nature reviews Urology. 2010;7(12):653-60. doi:10.1038/nrurol.2010.190.

2. Foxman B. Epidemiology of urinary tract infections: incidence, morbidity, and economic costs. The American journal of medicine. 2002;113 Suppl 1A:5s-13s.

3. Petersen I, Hayward AC. Antibacterial prescribing in primary care. The Journal of antimicrobial chemotherapy. 2007;60 Suppl 1:i43-7. doi:10.1093/jac/dkm156.

4. Ikaheimo R, Siitonen A, Heiskanen T, Karkkainen U, Kuosmanen P, Lipponen P et al. Recurrence of urinary tract infection in a primary care setting: analysis of a 1-year follow-up of 179 women. Clinical infectious diseases : an official publication of the Infectious Diseases Society of America. 1996;22(1):91-9.

5. Falagas ME, Kotsantis IK, Vouloumanou EK, Rafailidis PI. Antibiotics versus placebo in the treatment of women with uncomplicated cystitis: a meta-analysis of randomized controlled trials. The Journal of infection. 2009;58(2):91-102. doi:10.1016/j.jinf.2008.12.009.

6. Kronenberg A, Butikofer L, Odutayo A, Muhlemann K, da Costa BR, Battaglia M et al. Symptomatic treatment of uncomplicated lower urinary tract infections in the ambulatory setting: randomised, double blind trial. BMJ (Clinical research ed). 2017;359:j4784. doi:10.1136/bmj.j4784.

7. Gupta K, Hooton TM, Naber KG, Wullt B, Colgan R, Miller LG et al. International clinical practice guidelines for the treatment of acute uncomplicated cystitis and pyelonephritis in women: A2010 update by the Infectious Diseases Society of America and the European Society for Microbiologyand Infectious Diseases. Clinical infectious diseases : an official publication of the Infectious Diseases Society of America. 2011;52(5):e103-20. doi:10.1093/cid/ciq257.

8. Hasse B. HA HB, Egger M., Zanetti G., Marschall J., Mühlemann K., Harbarth S. Behandlung von unkomplizierten Harnwegsinfektionen. 2014. 9. Swiss Centre for Antibiotic resistance. 2018. www.anresis.ch. Accessed 23.09.2018. 10. Kronenberg A, Koenig S, Droz S, Muhlemann K. Active surveillance of antibiotic resistance prevalence in urinary tract and skin infections in the outpatient setting. Clinical microbiology and infection : the official publication of the European Society of Clinical Microbiology and Infectious Diseases. 2011;17(12):1845-51. doi:10.1111/j.1469-0691.2011.03519.x.

11. Hooton TM. Clinical practice. Uncomplicated urinary tract infection. The New England journal of medicine. 2012;366(11):1028-37. doi:10.1056/NEJMcp1104429.

12. Klingeberg A, Noll I, Willrich N, Feig M, Emrich D, Zill E et al. Antibiotic-Resistant E. coli in Uncomplicated Community-Acquired Urinary Tract Infection. Deutsches Arzteblatt international. 2018;115(29-30):494-500. doi:10.3238/arztebl.2018.0494.

13. Filippini M, Masiero G, Moschetti K. Socioeconomic determinants of regional differences in outpatient antibiotic consumption: evidence from Switzerland. Health policy (Amsterdam, Netherlands). 2006;78(1):77-92. doi:10.1016/j.healthpol.2005.09.009.

14. Achermann R, Suter K, Kronenberg A, Gyger P, Muhlemann K, Zimmerli W et al. Antibiotic use in adult outpatients in Switzerland in relation to regions, seasonality and point of care tests. Clinical microbiology and infection : the official publication of the European Society of Clinical Microbiology and Infectious Diseases. 2011;17(6):855-61. doi:10.1111/j.1469-0691.2010.03348.x.

15. Pluss-Suard C, Pannatier A, Kronenberg A, Muhlemann K, Zanetti G. Hospital antibiotic consumption in Switzerland: comparison of a multicultural country with Europe. The Journal of hospital infection. 2011;79(2):166-71. doi:10.1016/j.jhin.2011.05.028.

16. Hooton TM, Besser R, Foxman B, Fritsche TR, Nicolle LE. Acute uncomplicated cystitis in an era of increasing antibiotic resistance: a proposed approach to empirical therapy. Clinical infectious diseases : an official publication of the Infectious Diseases Society of America. 2004;39(1):75-80. doi:10.1086/422145.

17. ECDC. Surveillance of antimicrobial resistance in Europe 2017. Annual report of the European Antimicrobial Resistance Surveillance Network (EARS-Net) 2017.

18. Wagenlehner FM, Naber KG. Current challenges in the treatment of complicated urinary tract infections and prostatitis. Clinical microbiology and infection : the official publication of the European Society of Clinical Microbiology and Infectious Diseases. 2006;12 Suppl 3:67-80. doi:10.1111/j.14690691.2006.01398.x.

19. Peterson J, Kaul S, Khashab M, Fisher A, Kahn JB. Identification and pretherapy susceptibility of pathogens in patients with complicated urinary tract infection or acute pyelonephritis enrolled in a clinical study in the United States from November 2004 through April 2006. Clinical therapeutics. 2007;29(10):2215-21. doi:10.1016/j.clinthera.2007.10.008.

20. Hitzenbichler F, Simon M, Holzmann T, Iberer M, Zimmermann M, Salzberger B et al. Antibiotic resistance in $\mathrm{E}$. coli isolates from patients with urinary tract infections presenting to the emergency department. Infection. 2018;46(3):325-31. doi:10.1007/s15010-018-1117-5. 
21. Almomani BA, Hayajneh WA, Ayoub AM, Ababneh MA, AI Momani MA. Clinicalpatterns, epidemiology and risk factors of community-acquired urinary tract infection caused by extendedspectrum beta-lactamase producers: a prospective hospital case-control study. Infection. 2018;46(4):495-501. doi:10.1007/s15010-018-1148-y.

22. G. Bonkat RRB, F. Bruyère, T. Cai, S.E. Geerlings, B. Köves, S. Schubert, F. Wagenlehner. Urological Infections Guidelines 2018.

23. Johansen TE, Botto H, Cek M, Grabe M, Tenke P, Wagenlehner FM et al. Critical review of current definitions of urinary tract infections and proposal of an EAU/ESIU classification system. International journal of antimicrobial agents. 2011;38 Suppl:64-70. doi:10.1016/j.ijantimicag.2011.09.009.

24. Zhanel GG, Zhanel MA, Karlowsky JA. Oral Fosfomycin for the Treatment of Acute and Chronic Bacterial Prostatitis Caused by Multidrug-Resistant Escherichia coli. The Canadian journal of infectious diseases \& medical microbiology = Journal canadien des maladies infectieuses et de la microbiologie medicale. 2018;2018:1404813. doi:10.1155/2018/1404813.

25. Lipsky BA, Byren I, Hoey CT. Treatment of Bacterial Prostatitis. Clinical Infectious Diseases. 2010;50(12):1641-52. doi:10.1086/652861\%J Clinical Infectious Diseases.

26. Tenney J, Hudson N, Alnifaidy H, Li JTC, Fung KH. Risk factors for aquiring multidrug-resistant organisms in urinary tract infections: A systematic literature review. Saudi pharmaceutical journal: SPJ : the official publication of the Saudi Pharmaceutical Society. 2018;26(5):678-84. doi:10.1016/j.jsps.2018.02.023.

27. Garau J, Xercavins M, Rodriguez-Carballeira M, Gomez-Vera JR, Coll I, Vidal D et al. Emergence and dissemination of quinolone-resistant Escherichia coli in the community. Antimicrobial agents and chemotherapy. 1999;43(11):2736-41.

28. Alós JI, Serrano MG, Gómez-Garcés JL, Perianes J. Antibiotic resistance of Escherichia colifrom community-acquired urinary tract infections in relation to demographic and clinical data. Clinical Microbiology and Infection. 2005;11(3):199-203. doi:10.1111/j.1469-0691.2004.01057.x. 29. Ukah UV, Glass M, Avery B, Daignault D, Mulvey MR, Reid-Smith RJ et al. Risk factors for acquisition of multidrug-resistant Escherichia coli and development of community-acquired urinary tract infections. Epidemiologyand Infection. 2018;146(1):46-57. doi:10.1017/S0950268817002680. 30. Huttner A, Kowalczyk A, Turjeman A, Babich T, Brossier C, Eliakim-Raz N et al. Effect of 5-Day Nitrofurantoin vs Single-Dose Fosfomycin on Clinical Resolution of Uncomplicated Lower Urinary Tract Infection in Women: A Randomized Clinical TrialEffect of Nitrofurantoin vs Fosfomycin on Uncomplicated Lower UTI in WomenEffect of Nitrofurantoin vs Fosfomycin on Uncomplicated Lower UTI in Women. JAMA. 2018;319(17):1781-9. doi:10.1001/jama.2018.3627 \%J JAMA. 